\title{
Interaction between ACOT7 and LncRNA NMRAL2P via Methylation Regulates Gastric Cancer Progression
}

\author{
Huiqin Feng ${ }^{1}$ and Xiaojian Liu $^{2}$ \\ ${ }^{1}$ Department of Internal Medicine, Tongxiang Chinese Medicine Hospital, Tongxiang; \\ ${ }^{2}$ Department of Surgery, Tongxiang First People's Hospital, Tongxiang, China.
}

Purpose: Gastric cancer (GC) has a very poor prognosis when diagnosed at a late stage. Acyl-CoA thioesterase 7 (ACOT7) is a major isoform of the acyl coenzyme family that catalyzes the hydrolysis of fatty acyl-CoAs into unesterified free fatty acid and coenzyme A. The purpose of this study was to investigate the expression levels of ACOT7 in GC and mechanisms related therewith. Materials and Methods: Screening of systematic biology studies revealed ACOT7 as a key gene in GC, as well as involvement of the long non-coding RNA NMRAL2P in ACOT7 expression. In this study, GC tissues and adjacent tissue samples were obtained from 10 GC patients at the Department of Gastrointestinal Surgery. GES1 and SGC-7901 cells were collected and treated to silence ACOT7 and overexpress NMRAL2P. The expressions of ACOT7 and NMRAL2P were detected by real-time quantitative PCR and Western blot. Additionally, cell proliferation, apoptosis, migration, and invasion were examined.

Results: ACOT7 was upregulated in gastric tumor tissues and GC cell lines. ACOT7 gene silencing induced a less malignant phenotype and was closely correlated to reduced cell proliferation and migration, altered cell cycle, and increased apoptosis. Furthermore, NMRAL2P was downregulated in tumor tissues and GC cell lines. NMRAL2P overexpression induced a more malignant phenotype and significantly inhibited the expression of ACOT7. Importantly, NMRAL2P indirectly methylated ACOT7 by binding to DNMT3b, thereby suppressing ACOT7 expression.

Conclusion: NMRAL2P activation suppresses ACOT7 expression in GC. Thus, ACOT7 could be a promising target for the treatment of GC.

Key Words: Acyl-CoA thioesterase 7, gastric cancer, long non-coding RNA, methylation

\section{INTRODUCTION}

Gastric cancer (GC) is one of the most common malignant tumors of the alimentary system. Among all malignant tumors, the incidence of GC ranks fourth; its mortality ranks second. ${ }^{1}$ The pathogenesis of GC is a complex biological process involving multiple genes acting together to activate various tumorrelated pathways.

\footnotetext{
Received: November 19, 2019 Revised: March 13, 2020

Accepted: April 1, 2020

Corresponding author: Xiaojian Liu, MD, Department of Surgery, Tongxiang First People's Hospital, No. 136 Maodun West Road, Tongxiang 314500, China.

Tel: 86-0573-88218903, Fax: 86-0573-88218903, E-mail: liuxiaojian0123@sina.com

-The authors have no potential conflicts of interest to disclose.

(C) Copyright: Yonsei University College of Medicine 2020

This is an Open Access article distributed under the terms of the Creative Commons Attribution Non-Commercial License (https://creativecommons.org/licenses/ by-nc/4.0) which permits unrestricted non-commercial use, distribution, and reproduction in any medium, provided the original work is properly cited.
}

Fatty acyl-CoA is an important metabolite in lipid metabolism, which participates in many physiological processes, such as beta-oxidation, triacylglycerol, and phospholipid synthesis. ${ }^{2}$ Two enzyme families, acyl-CoA synthetases (ACSs) and acylCoA thioesterases (ACOTs), are required for the formation and degradation of fatty acyl-CoA, respectively. ${ }^{3}$ Human physiology involves more than 26 ACS enzymes and 13 ACOT enzymes, ${ }^{4,5}$ and recent studies have demonstrated the critical roles of these enzymes in GC. ${ }^{6}$

ACOT7 is the most widely studied member of the ACOT family. ${ }^{7}$ The enzyme is localized to the cytosol and is highly expressed in brain tissue and in the testis. ${ }^{8,9}$ ACOT7 is primarily involved in the hydrolysis of arachidonyl-CoA to arachidonic acid (AA) and CoA. AA is an important precursor molecule of eicosanoic acid (arachidic acid), which plays an important role in cell signal transduction, cell proliferation regulation, and metabolic enzymes activation..$^{10,11}$ Jung, et al. ${ }^{4}$ demonstrated that ACOT7 plays a critical role in cell cycle progression and 
might be a valuable target for radiotherapy and chemotherapy in lung cancer. However, the role of ACOT7 in GC has rarely been reported; therefore, the roles and mechanisms of ACOT7 in GC need to be elucidated.

The Cancer Genome Atlas (TCGA) is a collection of free, public molecular datasets accessible to cancer researchers worldwide. ${ }^{12}$ Meanwhile, the Gene Expression Omnibus (GEO) database is currently the largest fully open gene expression database; it includes a large number of gene microarrays and various forms of high-throughput gene sequencing data. ${ }^{13}$ We have found that differentially expressed genes and their tissue distribution characteristics are powerful tools for screening and identifying tumor markers.

Long non-coding RNA (lncRNA) is a non-protein coding RNA molecule with a length of more than 200 bases. ${ }^{14}$ LncRNAs play important roles in gene transcription and splicing regulation, post-transcriptional processing, chromosome remodeling, and protein-protein, protein-DNA, and protein-RNA interactions. ${ }^{15,16}$ LncRNA is differentially expressed in a variety of tumors, including GC, and its expression patterns affect the occurrence and development of tumors, migration, metastasis, relapse, and drug resistance, which make IncRNA a potential drug target. ${ }^{17}$ Importantly, the expression of IncRNA is diseaseand tissue-specific, which are ideal characteristics for diagnostic and prognostic markers. ${ }^{18}$ It has been confirmed that dysregulation of lncRNAs plays crucial roles in the regulation of oncogenes and tumor suppressor genes. ${ }^{19}$ The role of lncRNA NMRAL2P in carcinoma progression has been mentioned, ${ }^{20}$ although its role in GC has not been reported.

DNA methylation is an important epigenetic mechanism in many biological processes, including genome marking, $\mathrm{X}$ chromosome inactivation, normal cell development, and diffusion. ${ }^{21}$ DNA methylation primarily participates in histone acetylation and deacetylation, maintains DNA integrity and stability, and regulates gene expression. ${ }^{22}$ DNA methyltransferases (DNMTs), including DNMT1, DNMT2, and DNMT3, are an important family of enzymes that catalyze and maintain DNA methylation in epigenetics. ${ }^{23,24}$

In this study, we investigated the role of ACOT7 in linking IncRNA NMRAL2P and GC. ACOT7 was found to be up-regulated and inversely correlated with IncRNA NMRAL2P expression in GC. This may provide new clues on the expression levels and potential functions of ACOT7 in GC, as well as a potential mechanism for novel drug targets.

\section{MATERIALS AND METHODS}

\section{ACOT7 expression in GC from the TCGA database}

The TCGA database provides comprehensive cancer genomic datasets for researchers where data are available to search, download, and analyze. The Gene Expression Profiling Interactive Analysis database (GEPIA, http://gepia.cancer-pku. $\mathrm{cn} /)$ is an interactive webserver for analyzing gene expression data of tumors and normal tissues from TCGA and genotypetissue expression database. In this study, we searched the GEPIA database to examine ACOT7 expression in GC tissues and adjacent normal tissues.

\section{NMRAL2P (LncRNA) expression in GCs from the GEO database}

To evaluate gene expression in GC, a microarray dataset was obtained from the GEO database (http://www.ncbi.nlm.nih. gov/geo/). GSE84787 was downloaded from the website (https://www.ncbi.nlm.nih.gov/geo/geo2r/?acc=GSE84787), which was based on the Agilent GPL170077 platform (Agilent-039494 SurePrint G3 Human GE v2 8x60K Microarray 039381). Ten primary human advanced gastric carcinoma tissues and 10 noncancerous gastric tissues by high-density oligonucleotide microarray were included.

\section{Human tissue collection and qRT-PCR analysis}

GC tissues and adjacent tissue samples were obtained from 10 GC patients at the Department of Gastrointestinal Surgery at the Hangzhou Hospital of Traditional Chinese Medicine. The clinicopathologic characteristics of these patients are described in Supplementary Table 1 (only online). All patients had no history of radiotherapy, chemotherapy, hormone therapy, or other diseases that may interfere with the experimental results. This study was approved by the Institutional Review Board (IRB) of the Hangzhou Hospital of Traditional Chinese Medicine (IRB \#20180123), and written informed consent was obtained from all patients. The specimens were snap frozen with liquid nitrogen and stored at $-80^{\circ} \mathrm{C}$. Total RNA samples were extracted using TRIzol reagent (Invitrogen, Carlsbad, CA, USA) according to the manufacturer's instructions. Reverse transcription for cDNA synthesis and real-time quantitative PCR was performed using a SYBR PrimeScript RT Kit (Takara, Shiga, Japan) following the manufacturer's descriptions. The primer sequences for the tested genes (GenePharma, Shanghai, China) are provided in Supplementary Table 2 (only online). The reactions were performed over at least three independent runs for each sample. GAPDH was selected as a housekeeping gene, and the comparative cycle threshold $(\mathrm{Ct})$ method $\left(2^{-\Delta \Delta \mathrm{Ct}}\right)$ was used to analyze differences in each transcript. ${ }^{25}$

\section{Western blot assay}

Proteins from the tissues and cells were isolated using a protein extraction kit (Beyotime Biotechnology, Haimen, China), and the protein content was determined using a BCA Protein Assay Kit (Beyotime, Jiangsu, China). The protein samples were separated by SDS-PAGE gel electrophoresis (8-15\%) and transferred onto polyvinylidene difluoride (PVDF) membranes (EMD Millipore, Billerica, MA, USA). After $2 \mathrm{~h}$ of blocking with $5 \%$ bovine serum albumin in TBST buffer at room temperature, the PVDF membranes were individually incubated over- 
night at $4^{\circ} \mathrm{C}$ with the following primary antibodies: anti-ACOT7 (Abcam, Cambridge, UK) and anti-GAPDH (MultiSciences Biotech Co., Ltd, Hangzhou, China). After washing with TBST, the membranes were incubated at room temperature for $1 \mathrm{~h}$ with peroxidase-conjugated goat anti-rabbit immunoglobulin $\mathrm{G}$ antibodies (MultiSciences Biotech Co., Ltd) at a 1:2000 dilution. Protein expression was detected by the enhanced chemiluminescence method. Protein bands were imaged using Image Lab software (BIO-RAD, Hercules, CA, USA) and normalized with GAPDH as an internal control. The methods for determining protein content were described previously. ${ }^{26}$ The experiments were repeated at least three times.

\section{Cell culture}

Six cell lines were selected in this study: the immortalized normal human gastric mucosa cell line GES-1 and GC cell lines AGS, MGC-803, SGC-7901, MKN45, and BGC-823 cells purchased from the China Academia Sinica Cell Repository (Shanghai, China). GES-1, MGC-803, SGC-7901, MKN45, and BGC-823 were maintained in RPMI-1640 medium (Gibco, Carlsbad, CA, USA) containing $10 \%$ fetal bovine serum (FBS, Gibco). The AGS cells were cultured in F12 medium (Gibco) containing $10 \%$ FBS. All cell lines were cultured in an incubator at $5 \% \mathrm{CO}_{2}$ and $37^{\circ} \mathrm{C}$.

\section{Cell transfection}

SGC7901 cells were plated in 6-well plates at a concentration of $1 \times 10^{5}$ cells per well and cultured at $37^{\circ} \mathrm{C}$ with $5 \% \mathrm{CO}_{2}$ in RPMI1640 medium containing $10 \%$ FBS. Cells were transfected with specific GFP-tagged shRNA (Genepharma, Shanghai, China) targeting ACOT7 with Lipofectamine 2000 reagent (Invitrogen, Carlsbad, CA, USA). The vector (control) group of cells were treated with the same amount of nonsense RNA. After 5 days of transfection, the expression of green fluorescent protein (GFP) was observed under a fluorescence microscope.

\section{Cell proliferation assay}

SGC7901 cells were plated in 96-well plates at a concentration of $1 \times 10^{4}$ cells per well and incubated overnight. CCK8 reagent (Biotool, Shanghai, China) was added to each well, the plate was incubated for $3 \mathrm{~h}$ at $37^{\circ} \mathrm{C}$, and the colorimetric assay was measured at $450 \mathrm{~nm}$ using a microplate reader (Perkin-Elmer, Waltham, MA, USA).

\section{Cell cycle analysis by flow cytometry}

The cell cycle was detected by propidium iodide (PI) staining and flow cytometry. SGC 7901 cells from each group were seeded into 10-cm culture dishes at a concentration of $1 \times 10^{6}$ cells per dish and then incubated at $37^{\circ} \mathrm{C}$ with $5 \% \mathrm{CO}_{2}$ for $24 \mathrm{~h}$. The cell cycle was measured using a Cell Cycle Assay Kit (Becton Dickinson, Franklin Lakes, NJ, USA), according to the manufacturer's instructions. Apoptosis was measured using an Annexin-V (AV)-fluorescein isothiocyanate (FITC)/PI double staining kit (Shanghai Solja Technology, Shanghai, China). SGC7901 cells were cultured in 10-cm culture dishes at $1 \times 10^{6}$ cells per dish and incubated at $37^{\circ} \mathrm{C}$ with $5 \% \mathrm{CO}_{2}$ for $24 \mathrm{~h}$. The SGC7901 cells were collected and prepared into a single-cell suspension and then labeled according to the instructions provided by the AV/PI Apoptosis Detection Kit (Becton Dickinson). Flow cytometry (Cube6, Partec Group, Germany) was used to detect FITC at $488 \mathrm{~nm}$ and $515 \mathrm{~nm}$ and PI at excitation wavelengths of over $560 \mathrm{~nm}$.

\section{Scratch test}

From the logarithmic phase SGC7901 cell suspension, cells were seeded in a 6 -well culture plate $\left(5 \times 10^{5}\right.$ cells/well $)$. Each group was cultured with three parallel samples. The cells were incubated at $37^{\circ} \mathrm{C}$ with $5 \% \mathrm{CO}_{2}$ until the cells adhered and formed a single layer with cell fusion greater than $90 \%$. Then, a thin wound was created along the ruler on the surface of cells using a sterile pipette tip $(200 \mu \mathrm{L})$. The scratch line width was recorded under an inverted microscope (CX23, Olympus, Tokyo, Japan). After $24 \mathrm{~h}$ of culture, the well plate was removed for photography.

\section{Transwell assay}

Cell invasion and migration were detected using a Transwell system (EMD Millipore), as described previously. ${ }^{27}$ Briefly, $1 \times 10^{4}$ of cells in $200 \mu \mathrm{L}$ of serum-free medium were seeded in the upper chamber of the Transwell system, and the bottom of the chamber was filled with $600 \mu \mathrm{L}$ of medium containing $10 \%$ FBS. Each apical chamber was coated with diluted Matrigel (1:5; Becton Dickinson) and stored at $37^{\circ} \mathrm{C}$ for $3 \mathrm{~h}$. After $24 \mathrm{~h}$ of incubation at room temperature, the cells on the upper side of the filter membrane were removed. Crystal violet staining was performed at $25^{\circ} \mathrm{C}$ for 15 minutes, and an inverted light microscope (magnification, $\times 20$ ) was used to count the number of cells adhering to the submembrane.

\section{NMRAL2P overexpression in cells}

Overexpressed DNA transfection was used to upregulate NMRAL2P expression levels. The cDNA sequence of NMRAL2P was inserted into the pcDNA3.1 expression vector (Invitrogen, Shanghai, China) as previously described. ${ }^{28}$ The SGC7901 cells were transfected with NMRAL2P plasmid DNA or empty vector as a control (GenePharma, Shanghai, China) using Lipofectamine 2000 reagent according to the manufacturer's instructions.

\section{Methylation-specific polymerase chain reaction}

DNA was treated with sodium bisulfite to convert unmethylated cytosines into uracil. After removing sodium bisulfate and completing chemical transformation, the modified DNA was used as a template for PCR. Primers were designed to distinguish methylation and unmethylation alleles after sodium bisulfate treatment and to identify sodium bisulfate-modified 
DNA (compared with unmodified DNA).

\section{Luciferase reporter assay}

SGC7901 cells were plated in 24-well plates for $12 \mathrm{~h}$. Subsequently, ACOT7 promoter sequences were introduced into the pGL3 vector (Promega Cooperation, Madison, WI, USA) for ACOT7 promoter transcriptional activity assay. Reporter plasmids (100 ng; EMD Millipore) containing TOP-Flash or FOPFlash luciferase reporter plus 1 ng of pRL-TK Renilla plasmid were co-transfected using Lipofectamine 2000 (Invitrogen, Carlsbad, CA, USA; Thermo Fisher Scientific, Inc., Hongkong, China). After $48 \mathrm{~h}$ of transfection, luciferase activity was measured using a Dual Luciferase Reporter assay kit (Promega Cooperation) according to the manufacturer's protocol.

\section{DNA methylation}

Genomic DNA was extracted from GES-1 cells treated with the NMRAL2P expression lentivirus, silencing, 5-aza-2'-deoxycytidine (5-aza-dC, Sigma, Saint Louis, MO, USA) and their control group cells. Sodium bisulfate from the EZ DNA Methylation-Gold TM Kit (Qiagen, Valencia, Canada) was added into the DNA samples to detect the methylation of ACOT7 promoter using the methylation-specific PCR (MSP) method.

\section{Statistical analysis}

All values are expressed as a mean \pm standard deviation (SD). The F test was used to analyze the homogeneity of variance among quantitative data groups, the $t$ test and non-parametric test were used to compare the data between groups, and the threshold of significance was selected as a $p$ value $<0.05$. Quantitative data analysis was performed in Graph Pad Prism 7 (GraphPad Software, Inc., La Jolla, CA, USA) and SPSS 23.0 (IBM Corp., Armonk, NY, USA).

\section{RESULTS}

\section{Expression analysis of ACOT7 using TCGA}

A total of $408 \mathrm{GC}$ samples and 211 adjacent normal tissues samples were collected from the TCGA database. The results revealed that ACOT7 expression was significantly increased in the GC group, compared to the adjacent normal tissue group (Fig. 1A). Similarly, the survival analysis results from KaplanMeier plots depicted the association between gene expression levels and clinical parameters on patient survival: high ACOT7 expression was associated with worse overall survival (Fig. 1B). The $\log$-rank $p$ value revealed the statistical significance of the observed patterns. Overall survival curves were generated by setting median ACOT7 expression as the cutoff.

\section{Expression of ACOT7 in GC tissues and cell lines}

The results showed that ACOT7 expression levels were significantly increased in all examined carcinoma tissues, compared with para-carcinoma tissues (Fig. $1 \mathrm{C}$ and D). The expression levels of ACOT7 in the normal human gastric epithelial cell line GES-1 and a group of GC cell lines were evaluated. ACOT7 had higher expression in GC cell lines than the GES- 1 cells at both the protein and mRNA level (Fig. 1E and F).

\section{ACOT7 silencing inhibits proliferation and cell cycle progression and promotes apoptosis of GC cells} In order to determine the role of ACOT7 in GC, we observed the effect of ACOT7 silencing on the GC cell line SGC7901. The efficiency of ACOT7 shRNA was confirmed by fluorescence microscopy, Western blots, and qRT-PCR (Fig. 2A and B). CCK8 assay demonstrated that ACOT7 silencing significantly inhibited the viability of SGC7901 cells (Fig. 2C). The effect of ACOT7 silencing was further investigated by studying the cell cycle and apoptosis of SGC7901 cells using flow cytometry. The results showed that ACOT7 silencing markedly increased the number of cells in the $\mathrm{Gl}$ and $\mathrm{S}$ phase, and decreased the number of cells in G2 (Fig. 2D). A cell apoptosis assay measuring AV/PI staining revealed that silencing ACOT7 expression promotes the cell apoptosis rate, compared to the vector group (Fig. 2E). Together these results suggested that ACOT7 might stimulate cell proliferation, promote cell cycle progression, and inhibit apoptosis.

\section{ACOT7 silencing inhibits migration and invasion in GC cells}

The transwell assay revealed that the number of invaded cells in the vector group were significantly increased when compared to the ACOT7-silenced group (Fig. 2F). In the woundhealing assay, the migration rate of ACOT7 shRNA-transfected cells was significantly inhibited when compared to vector group cells (Fig. 2G). These findings indicated that ACOT7 could enhance the migration and invasion abilities of GC cells in vitro.

\section{Expression of IncRNA NMRAL2P in GC}

The GEO database results revealed that NMRAL2P expression was markedly decreased in the GC tissues, compared with the adjacent normal tissues (Fig. 3A). The part of the data downloaded from the GEO database are provided in Supplementary Table 3 (only online). The qRT-PCR results showed that NMRAL2P expression levels were significantly decreased in all examined carcinoma tissues (Fig. 3B), compared to para-carcinoma tissues. NMRAL2P mRNA was expressed at lower levels in all examined GC cell lines, compared to the GES-1 cells (Fig. 3C).

\section{Overexpression of NMRAL2P inhibits cell proliferation, cell cycle progression, cell migration, and invasion and promotes cell apoptosis in GC cells The qRT-PCR results of NMRAL2P overexpression in the GC cell line SGC7901 are shown in Fig. 4A. CCK8 assay demon- strated that NMRAL2P overexpression significantly inhibited the viability of SGC7901 cells (Fig. 4B). The flow cytometry re- sults revealed that NMRAL2P overexpression markedly in-}




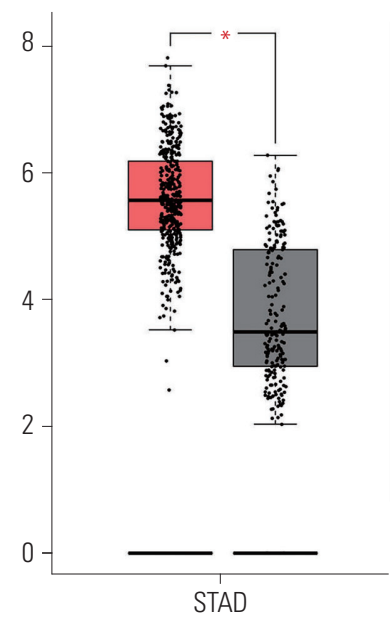

A $\quad[\operatorname{num}(\mathrm{T})=408 ; \operatorname{num}(\mathrm{N})=211]$

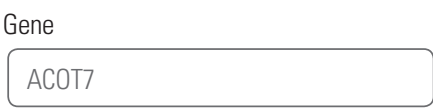

Input a gene symbol or id.

$\left|\log _{2} \mathrm{FC}\right|$ cutoff:

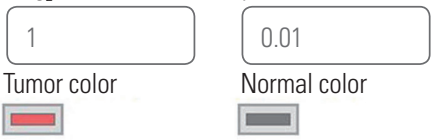

$\square$

Overall survival

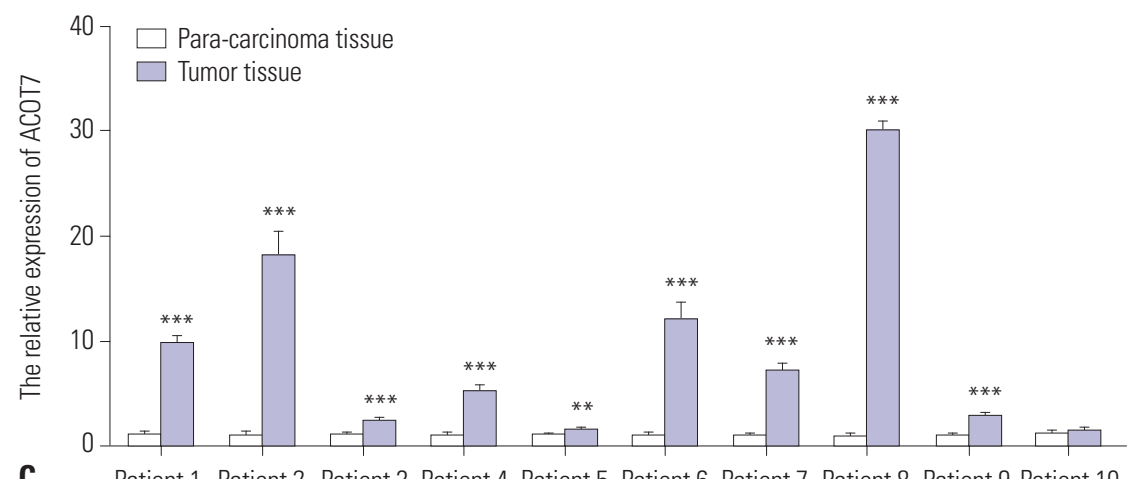

C Patient 1 Patient 2 Patient 3 Patient 4 Patient 5 Patient 6 Patient 7 Patient 8 Patient 9 Patient 10
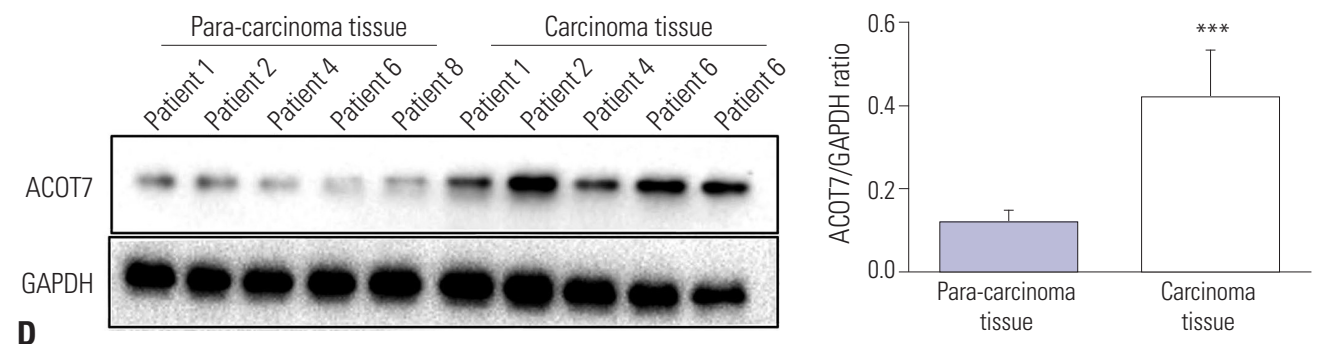

D

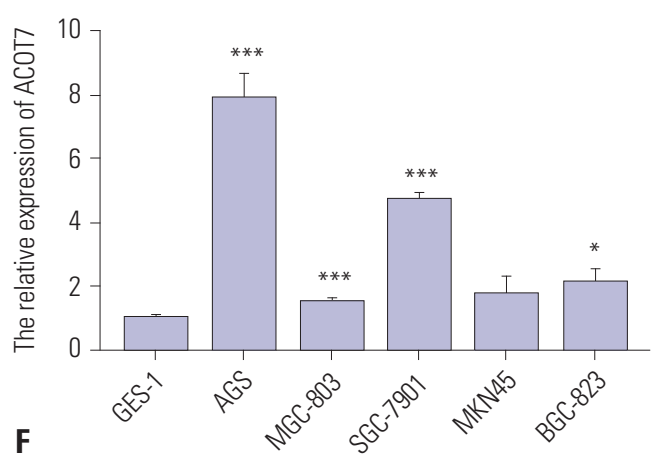

Fig. 1. The expression of $\mathrm{ACOT7}$ in GC. (A) Boxplot showing the relative expression of ACOT7 in normal and GC samples $\left({ }^{*} p<0.05\right)$. (B) Kaplan-Meier curves of overall survival in patients with high or low ACOT7 expression in TCGA-GC. (C) The relative expression of ACOT7 in GC tissues by qRT-PCR assay. ${ }^{* *}$ Compared with the para-carcinoma tissues group, $p<0.01,{ }^{* * *}$ Compared with the para-carcinoma tissues group, $p<0.001$. (D) Western blot analysis of the expression of ACOT7 in GC tissues. ${ }^{* *}$ Compared with para-carcinoma tissues group, $p<0.001$. (E) Western blot analysis of the expression of ACOT7 protein in the normal human gastric epithelial cell line GES-1 and a panel of GC cell lines. (F) The relative expression of ACOT7 mRNA normalized to GAPDH in GES- 1 cells and a panel of GC cell lines. ${ }^{*}$ Compared with GES- 1 cells, $p<0.05,{ }^{* * *}$ Compared with para-carcinoma tissues group, $p<0.001$. STAD: stomach adenocarcinoma; ACOT7, acyl-CoA thioesterase 7; GC, gastric cancer; TCGA, The Cancer Genome Atlas. 
creased the number of cells in the G1 and S phase, and decreased the number of cells in G2 (Fig. 4C). AV/PI staining revealed that overexpression NMRAL2P promoted the cell apoptosis rate, compared to the control group (Fig. 4D). Together these results suggested that NMRAL2P might inhibit cell proliferation and cell cycle progression and promote cell apoptosis.

In the wound healing assay, the migration rate of the NMRAL2P overexpression-group was markedly lower than that

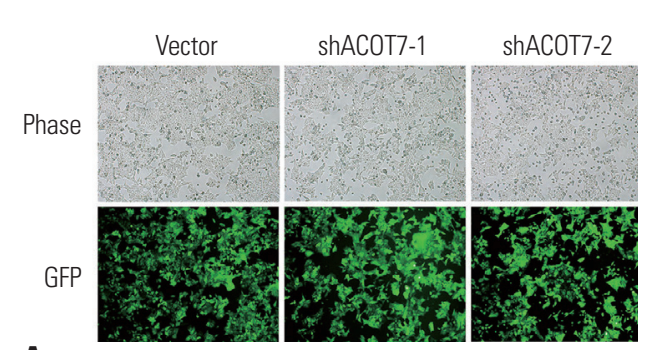

A

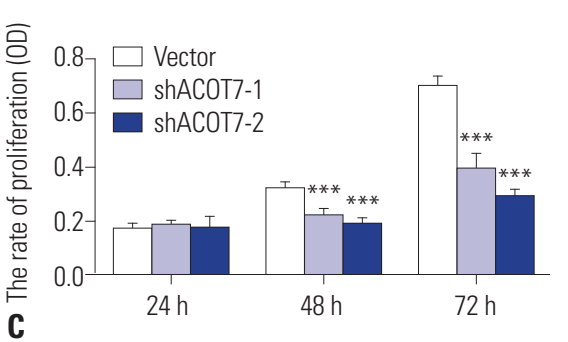

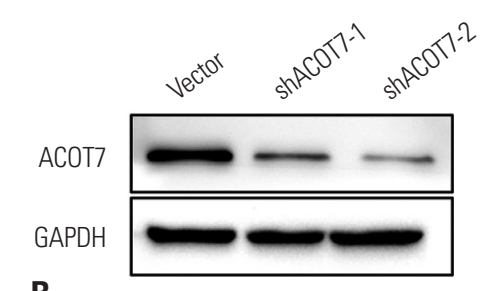

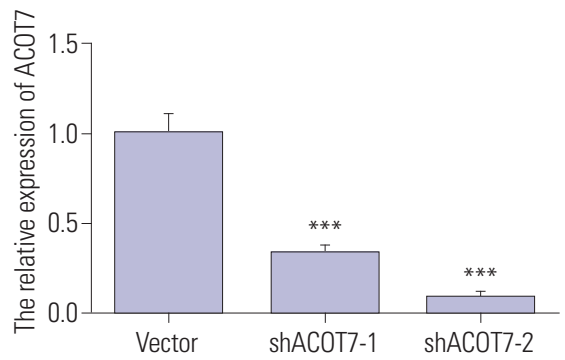

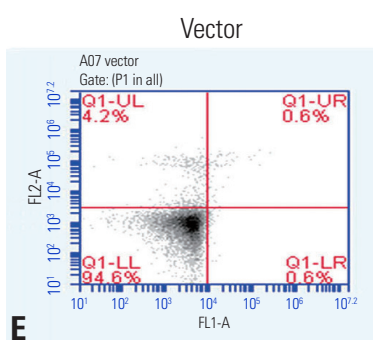
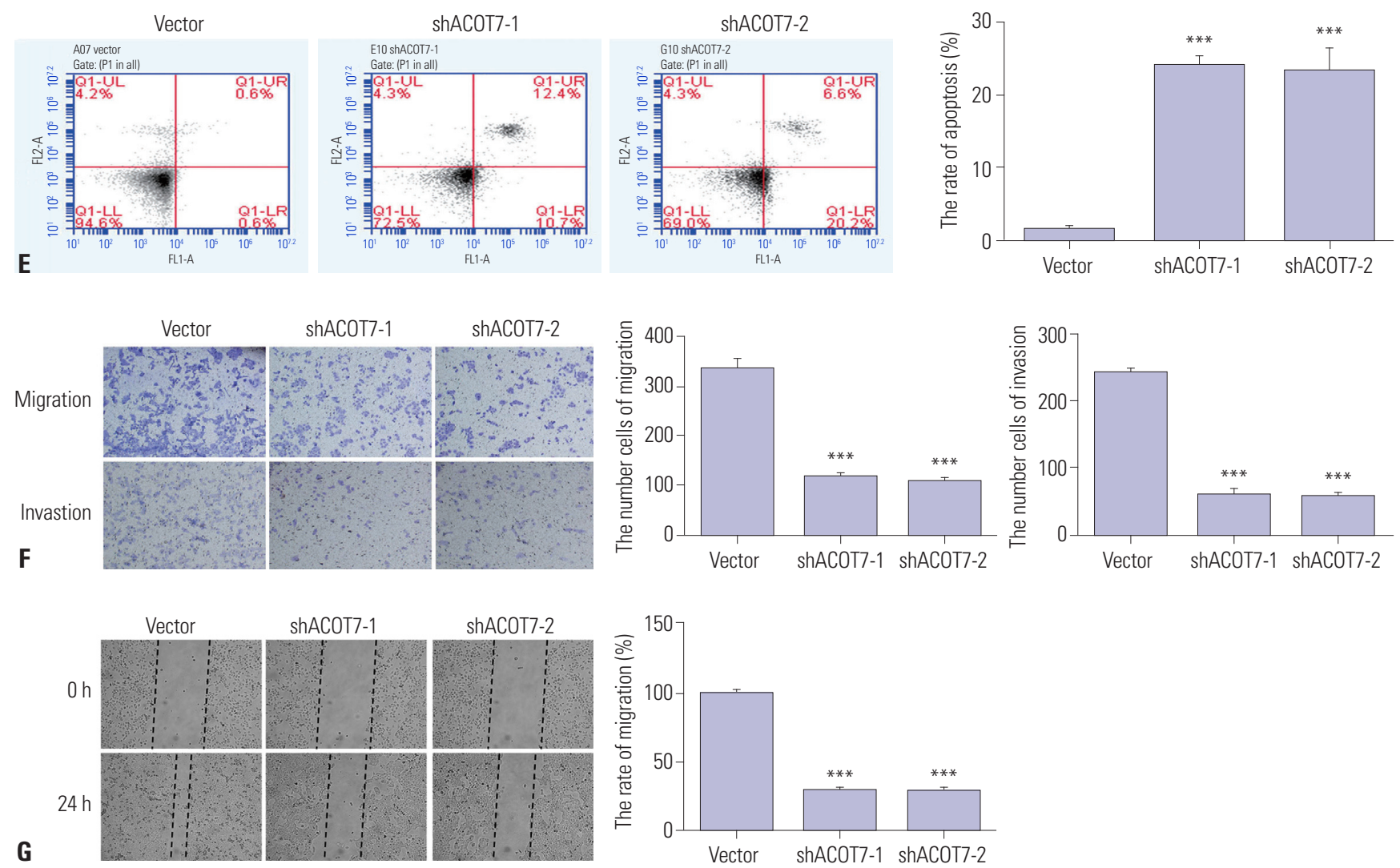

Fig. 2. The effects of ACOT7 silencing on the proliferation, cell cycle progression, apoptosis, migration, and invasion of SGC7901 cells. (A) The expression of ACOT7-1 and ACOT7-2 after transfection under a fluorescence microscope using GFP $(\times 200)$. (B) The expression of ACOT7-1 and ACOT7-2 proteins after transfection upon Western blot assay and PCR. (C) The proliferation rate of SGC7901 after transfection. (D) Cell cycle progression of SGC7901 after transfection. (E) Flow cytometry analysis of apoptosis of SGC7901 cells after transfection by flow cytometry. (F) Cell migration and invasion of SGC7901 cells after transfection using crystal violet staining $(\times 200)$. (G) The scratch test was performed by scratching a confluent monolayer of cells with a 200-uL pipette tip. The migration of SGC7901 cells was analyzed after 24 hours. ${ }^{* * *}$ Compared with empty vector, $p<0.001$. GFP, green fluorescent protein; ACOT7, acyl-CoA thioesterase 7. 

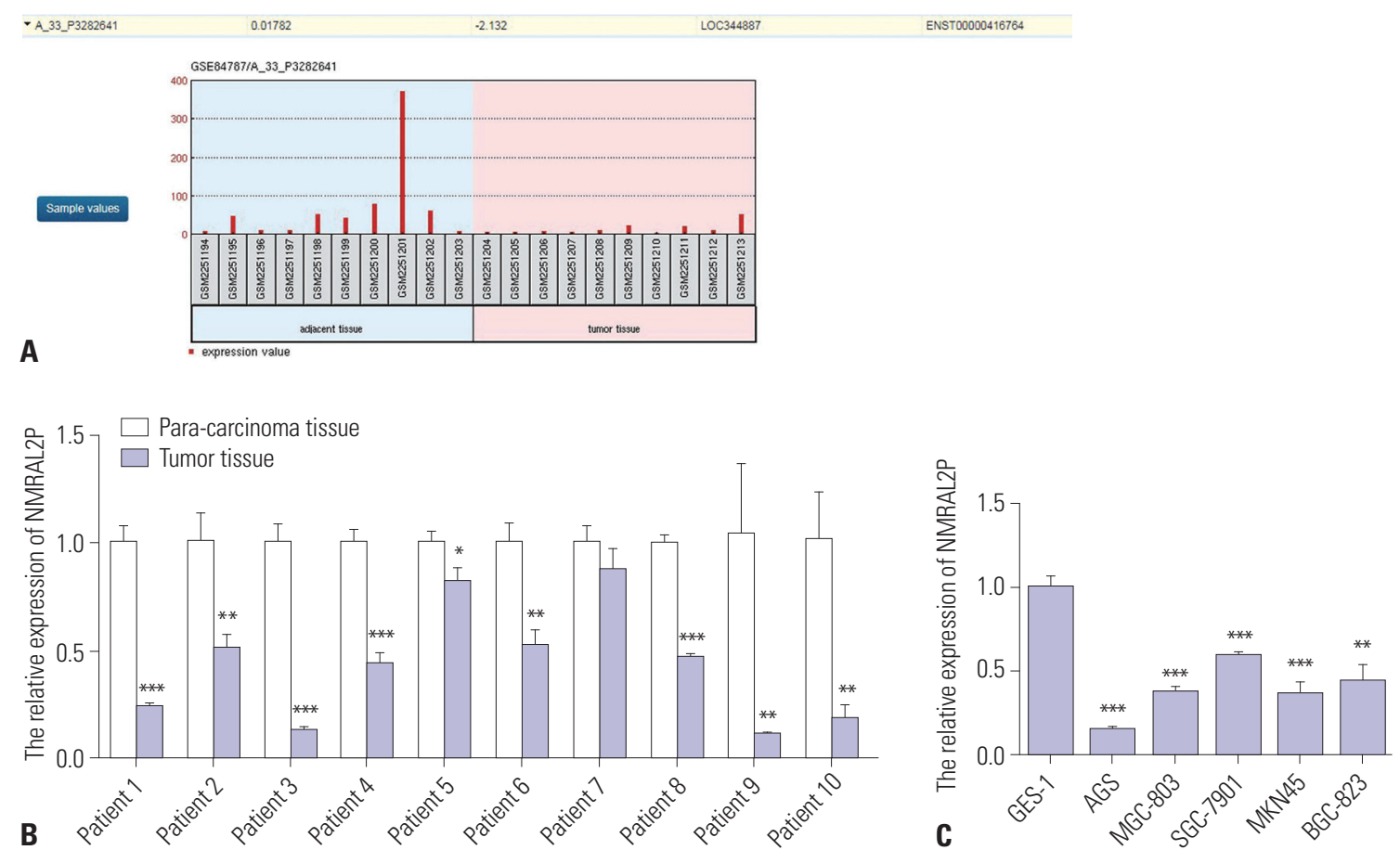

Fig. 3. The expression of IncRNA NMRAL2P in gastric cancer. (A) The expression of NMRAL2P in normal and GC samples from the GEO database. (B) The relative expression of NMRAL2P mRNA in GC tissues. ${ }^{*}$ Compared with para-carcinoma tissues group, $p<0.05$, ${ }^{*}$ Compared with para-carcinoma tissues group, $p<0.01,{ }^{* * *}$ Compared with para-carcinoma tissues group, $p<0.001$. (C) The relative expression of NMRAL2P mRNA in GES- 1 cells and a panel of GC cell lines. ${ }^{* *}$ Compared with GES-1 cells, $p<0.01$, ${ }^{* * *}$ Compared with GES-1 cells, $p<0.001$. IncRNA, long non-coding RNA; GC, gastric cancer.

of the control group cells (Fig. 4E). The results revealed that the number of invaded cells in the control group were significantly increased when compared to the NMRAL2P overexpression group (Fig. 4F). Collectively, these findings suggested that NMRAL2P in GC inhibits GC cell proliferation, migration, and invasion.

\section{Repression of ACOT7 by lncRNA NMRAL2P}

Many lncRNAs inhibit the occurrence and development of cancer through epigenetic regulation of gene expression. ${ }^{29} \mathrm{~A}$ panel of IncRNAs from the GEO database indicated that lncRNA NMRAL2P was poorly expressed in GC. By investigating the regulatory relationship between NMRAL2P and ACOT7, we found that NMRAL2P overexpression inhibited the expression of ACOT7 in SGC7901 cells (Fig. 5A). To further understand the molecular basis of NMRAL2P-mediated ACOT7 repression, we examined whether ACOT7 is directly regulated by NMRAL2P. Bioinformatics analysis showed that methylation of the ACOT7 promoter decreased significantly in gastric tumor samples (Fig. 5B).

NMRAL2P upregulates DNMT3b for DNA methylation of ACOT7 promoter to suppress ACOT7 gene expression Recent studies have indicated that lncRNAs are important players in the regulation of DNA methylation. ${ }^{30}$ Therefore, we speculated that NMRAL2P may regulate the methylation of ACOT7 by binding to DNMT. MSP is a method used to analyze the DNA methylation patterns in CpG islands, and it showed that ACOT7 was methylated in gastric para-carcinoma tissues and GES- 1 cells and unmethylated in the gastric carcinoma tissues and SGC7901 cells (Fig. 5C). We transfected SGC7901 cells with unmethylated or completely methylated ACOT7pGL3 construct with a pRL-TK plasmid as a control for transfection efficiency. Methylation of the ACOT7-pGL3 plasmid resulted in a $>10$-fold inhibition of reporter activity (Fig. 5D).

To determine whether this activation was related to changes in the methylation of ACOT7 promoter, DNA was isolated from drug-treated GES- 1 cells and analyzed by MSP. GES- 1 cells were treated with the methylation inhibitor 5 -aza-dC at varying concentrations and over different lengths of time in an attempt to induce ACOT7 expression. 5-aza-dC treatment resulted in different degrees of partial demethylation of the ACOT7 promoter in a concentration-dependent manner (Fig. 5E). Consistent with our methylation analysis, induction of ACOT7 protein and mRNA levels were observed (Fig. 5F). Similarly, GES-1 cells were treated with the methylation inhibitor 5-aza$\mathrm{dC}$ for varying lengths of time. DNA methylation of ACOT7 was decreased (Fig. 5G) in a time-dependent manner, whereas ACOT7 expression increased (Fig. $5 \mathrm{H}$ ).

To better study the methylation-dependent mechanism of ACOT7, the expression levels of the main DNA methyltransferases DNMT1, DNMT3a, and DNMT3b were further evaluated by RNA interference. In cells incubated with cDNA to NMRAL2P, a notable increase in DNMT3b expression was observed, and DNMT3b expression was significantly decreased after treatment with NMRAL2P shRNA (Fig. 5I). Meanwhile, the expres- 

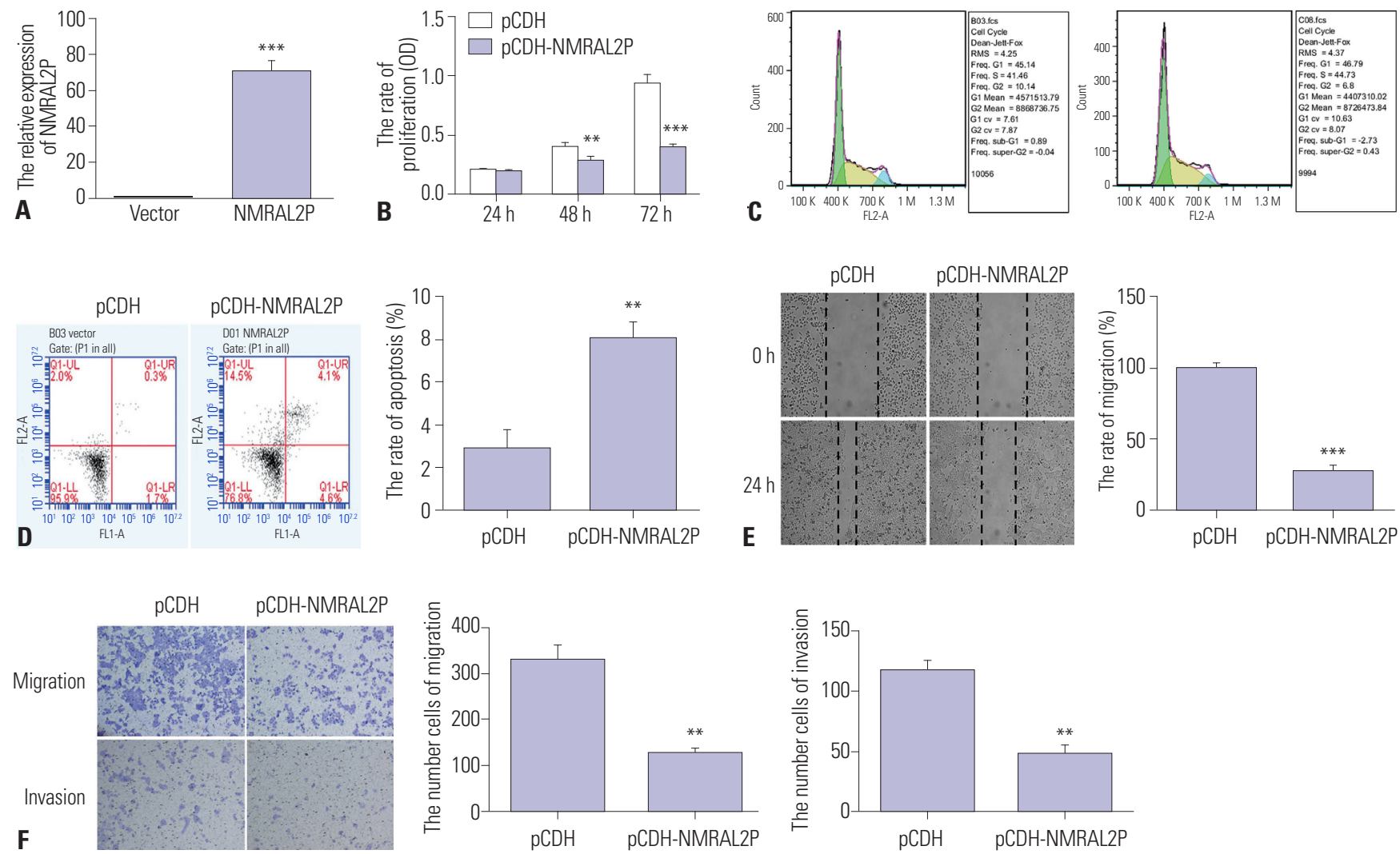

Fig. 4. The effects of NMRAL2P overexpression on cell proliferation, cycle, apoptosis, migration, and invasion in SGC7901 cells. (A) The overexpression of NMRAL2P after transfection was confirmed by qRT-PCR assay. (B) The proliferation rate of SGC7901 after transfection. (C) The cell cycle progression of SGC7901 after transfection. (D) The cell apoptosis of SGC7901 cells after transfection. (E) The scratch test was conducted to examine the migration of SGC7901 cells after transfection. (F) The cell migration and invasion of SGC7901 cells after transfection using crystal violet staining $(\times 200)$. ${ }^{* *}$ Compared with empty vector, $p<0.01,{ }^{* * *}$ Compared with empty vector, $p<0.001$.

sion levels of ACOT7 were increased after treatment with DNMT3b siRNA (Fig. 5J). The results indicated that NMRAL2P might suppress ACOT7 expression by regulating methylation via binding to DNMT3b.

\section{DISCUSSION}

GC is a common malignant tumor with a high rate of mortality. Although the strategies of surgery, chemotherapy, and radiotherapy have continuously improved, outcomes for patients with advanced GC are not ideal. Therefore, it is necessary to explore sensitive and effective therapeutic targets. The present study investigated the role of ACOT7 in GC using tumor tissues and GC cell lines. The lncRNA NMRAL2P was found to regulate GC progression through inhibition of ACOT7 by methylation.

TCGA analysis revealed that ACOT7 is highly expressed in GC. Similar results were also obtained in gastric carcinoma tissues and the majority of GC cell lines tested. ACOT7 silencing in the GC cell line SGC7901 decreased cell proliferation rate, migration, and invasion; promoted apoptosis; and arrested the cell cycle at the G1 and S phase. These findings indicated that ACOT7 exerts carcinogenic effects by promoting GC cell proliferation and migration.

The increased expression of ACOT7 in GC may be related to genomic alterations, epigenetic modifications, or subcellular location changes. DNA methylation is the most common factor leading to the acceleration of cancer progression ${ }^{31,32}$ and an important epigenetic modification. Hypomethylation of DNA promotes the development of cancer to a certain extent by activating genes with carcinogenic potential. ${ }^{32,33}$ Similarly, hypomethylation of oncogenes or hypermethylation of tumor suppressors is an important mechanisms of tumorigenesis. ${ }^{34,35}$ In the present study, analysis of the ACOT7 promoter region revealed that DNA methylation was markedly reduced in gastric carcinoma tissues and SGC7901 cells. Moreover, the methylation inhibitor 5-aza-dC concentration- and time-dependently increased ACOT7 expression in GES-1 cells, confirming the regulatory role of methylation.

Overexpression of the novel lncRNA NMRAL2P was found to downregulate ACOT7. Analysis of GSE84787 in the GEO database revealed that NMRAL2P is downregulated in GC; this finding was supported by the qRT-PCR-based comparison of NMRAL2P expression in gastric tumor tissues and various GC cell lines with that in para-carcinoma tissues and the normal 


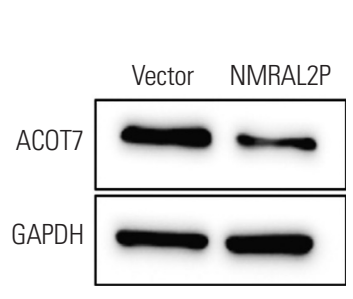

A

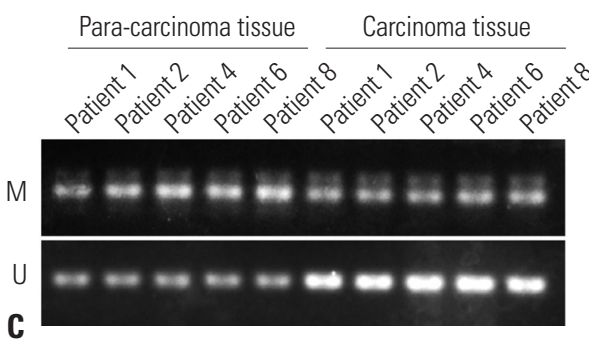

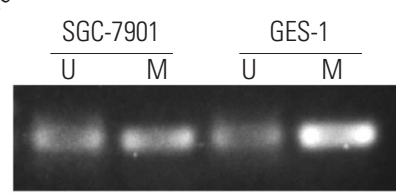

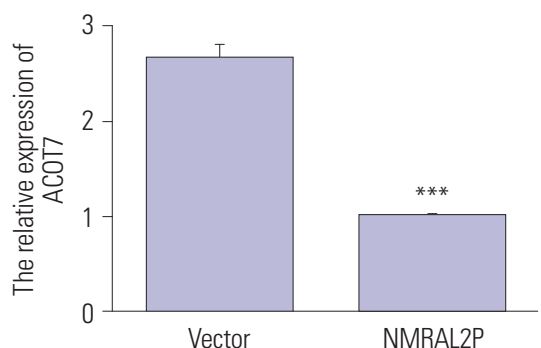

NMRAL2P
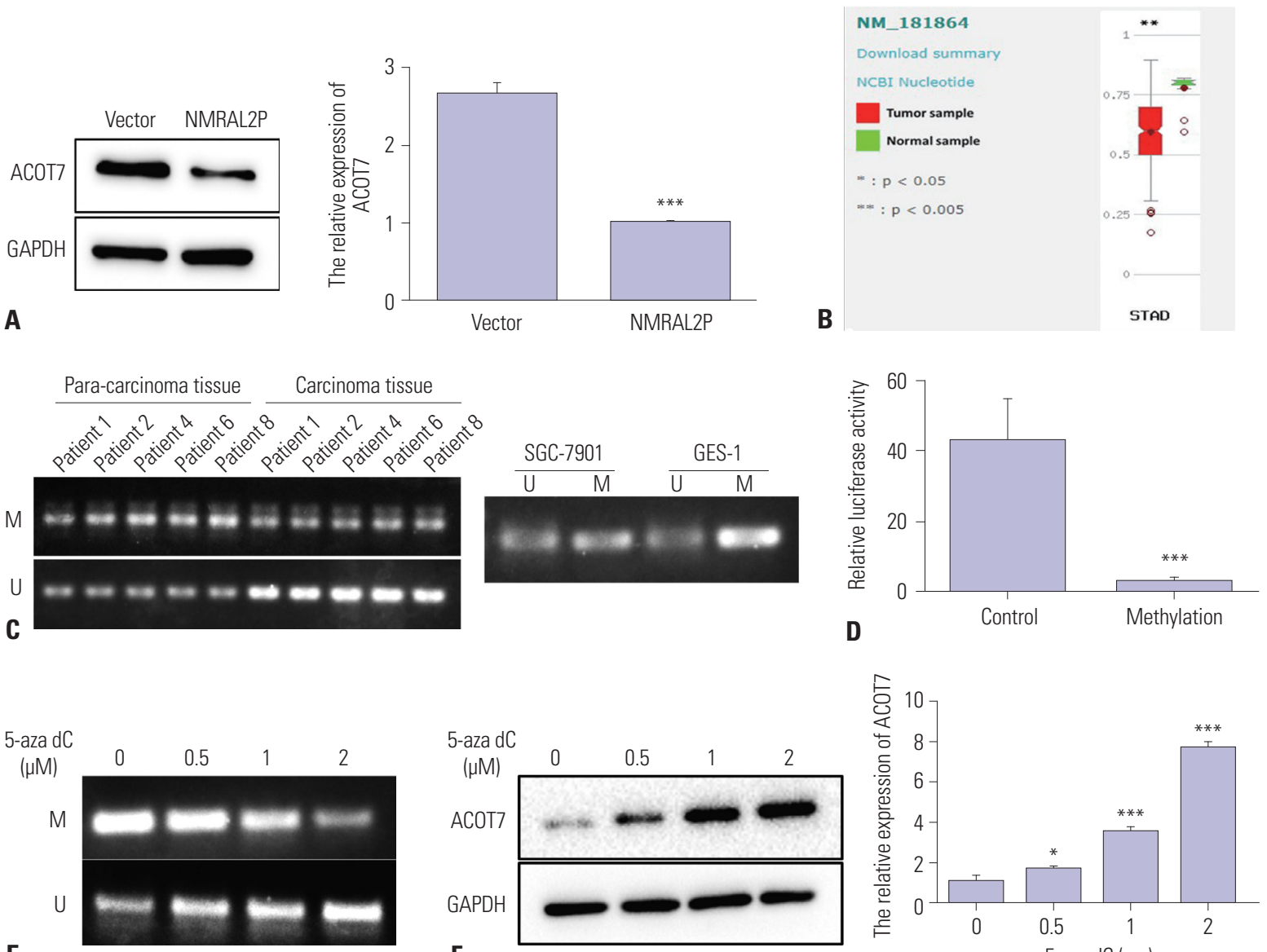

$\mathbf{E}$

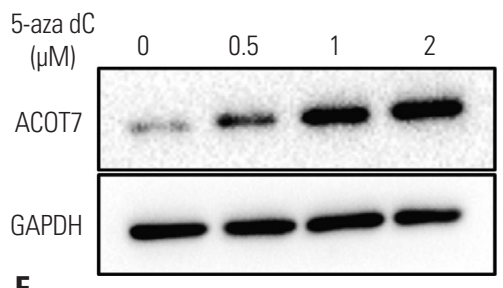

$\mathbf{F}$
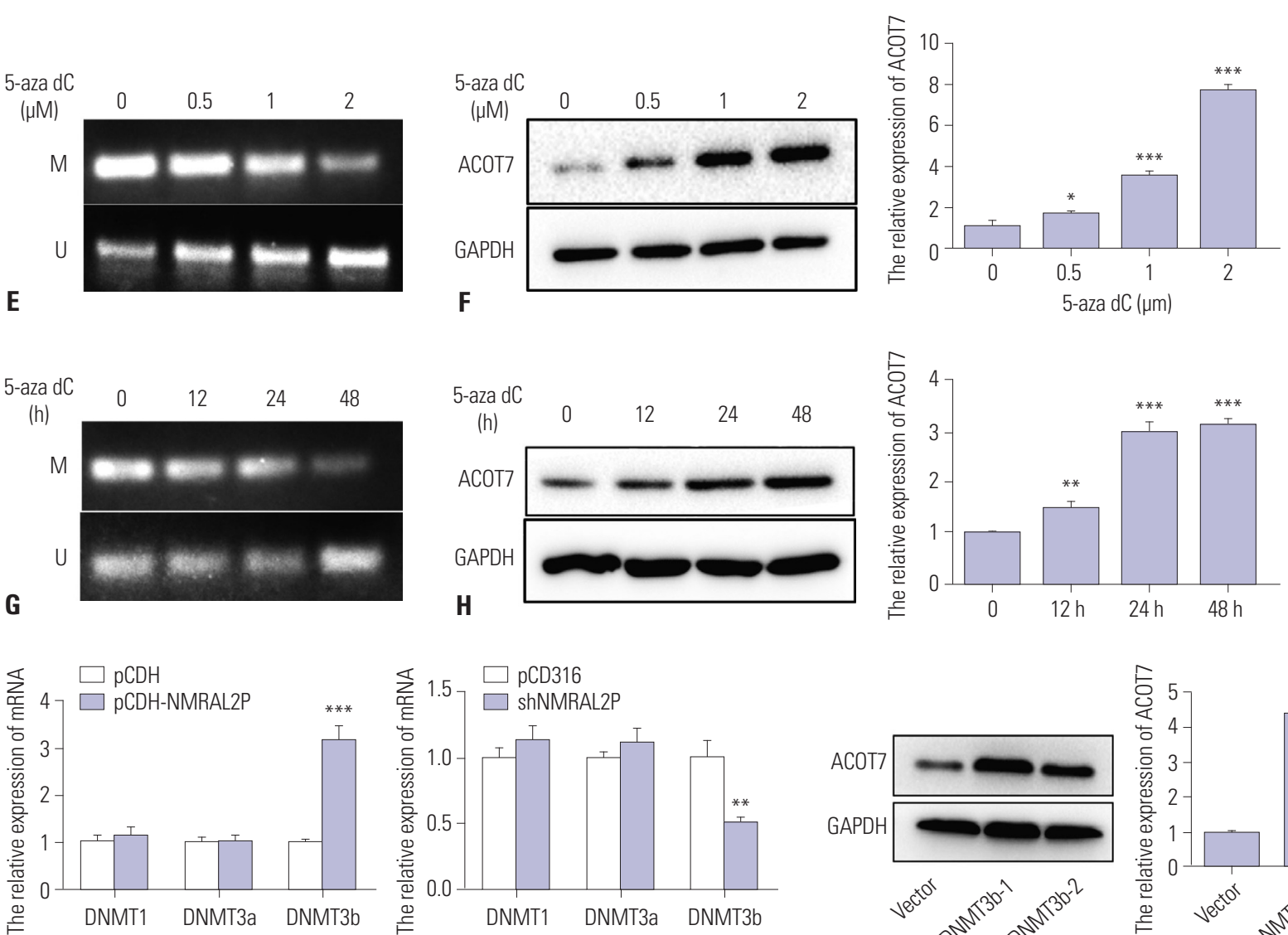

Fig. 5. NMRAL2P upregulates DNMT3b for DNA methylation of ACOT7 promoter to suppress ACOT7 expression. (A) The expression of ACOT7 after NMRAL2P overexpression on Western blot and qRT-PCR assays. (B) Boxplot showing the relative expression of ACOT7 in normal and GC samples. (C) The methylation of ACOT7 promotor in GC tissues and GC cells SGC7901 and normal cells GES-1 by MSP assay. (D) The methylation of ACOT7-pGL3 in SGC7901 by dual-luciferase assay. (E) GES-1 cells were treated with 5 -aza-dC $(0.5,1,2 \mu \mathrm{M})$ and methylated ACOT7 was reduced by MSP. (F) In GES-1 cells, the protein and mRNA levels of ACOT7 were analyzed by Western blot analysis and qRT-PCR, respectively, after 5-aza-dC $(0.5,1,2 \mu \mathrm{M})$ treatment for $48 \mathrm{~h}$. (G) GES1 cells were treated with 5 -aza-dC $(2 \mu \mathrm{M})$ for 12,24 , and $48 \mathrm{~h}$, and methylated ACOT7 was reduced by MSP. (H) In GES-1 cells, the protein and mRNA levels of ACOT7 were analyzed by Western blot analysis and qRT-PCR assay after 5-aza-dC treatment for 12, 24, and $48 \mathrm{~h}$. (I) The relative expression of DNMT1, DNMT3a, and DNMT3b after NMRAL2P overexpression and silencing by qRT-PCR assay. (J) The protein and mRNA levels of ACOT7 were analyzed by Western blot analysis and qRT-PCR assay after DNMT3b silencing in GES-1 cells. ${ }^{*}$ Compared with control, $p<0.05,{ }^{* *}$ Compared with control, $p<0.01$, ${ }^{* *}$ Compared with control, $p<0.001$. ACOT7, acyl-CoA thioesterase 7; GC, gastric cancer; MSP, methylation-specific PCR; $\mathrm{U}$, unmethylation; $\mathrm{M}$, methylation. 
gastric cell line GES-1. Further, NMRAL2P overexpression in SGC7901 cells by cDNA transfection inhibited cell proliferation, migration, and invasion; promoted apoptosis; and arrested the cell cycle at the $\mathrm{Gl}$ and $\mathrm{S}$ phase. These findings indicated that NMRAL2P plays a tumor suppressive role by inhibiting GC cell proliferation and migration. LncRNAs are associated with the occurrence, metastasis, prognosis, and drug resistance of GC. ${ }^{36,37}$ We speculate that the tumor suppressive effect observed for lncRNA NMRAL2P in this study is likely exerted via methylation of ACOT7 through binding to DNMT3b, one of the main enzymes involved in DNA methylation. Despite the clinical implications, some limitations must be noted. First, the study included small sample sizes, so more clinical samples need to be collected for further study. Second, the exact mechanism of the interaction between ACOT7 and IncRNA NMRAL2P require further exploration.

Overall, in this study, we discovered that ACOT7 expression is markedly increased in GC tissues and cell lines and that the loss of ACOT7 expression is correlated with cell proliferation, cell cycle, apoptosis, migration, and invasion. RNAi inhibited the expression of ACOT7, increased the apoptosis of GC cells, affected cell cycle changes, and reduced the growth and tumorigenicity of GC cells. Furthermore, our results indicated that NMRAL2P exerts a potential inhibitory effect on ACOT7 by methylation via the combination of DNMT3b and ACOT7 promoter. Therefore, constitutive NMRAL2P activation was possible because of a lack of ACOT7 expression in GC cells. The results indicated that ACOT7 may be a critical therapeutic target for patients with GC, providing a new perspective for future research.

\section{ACKNOWLEDGEMENTS}

This study was supported by the National Natural Science Foundation of China (Grant No. 201801014).

\section{AUTHOR CONTRIBUTIONS}

Conceptualization: Xiaojian Liu and Huiqin Feng. Data curation: Huiqin Feng. Formal analysis: Xiaojian Liu and Huiqin Feng. Funding acquisition: Xiaojian Liu. Investigation: Huiqin Feng. Methodology: Huiqin Feng. Project administration: Xiaojian Liu. Resources: Xiaojian Liu. Software: Xiaojian Liu and Huiqin Feng. Supervision: Xiaojian Liu. Validation: Xiaojian Liu and Huiqin Feng. Visualization: Huiqin Feng. Writing —original draft: Huiqin Feng. Writing — review \& editing: Xiaojian Liu. Approval of final manuscript: all authors.

\section{ORCID iDs}

\section{Huiqin Feng https://orcid.org/0000-0002-9149-272X}

Xiaojian Liu https://orcid.org/0000-0002-6137-3869

\section{REFERENCES}

1. Gan J, Ke X, Jiang J, Dong H, Yao Z, Lin Y, et al. Growth hormonereleasing hormone receptor antagonists inhibit human gastric cancer through downregulation of PAK1-STAT3/NF- $\kappa B$ signaling. Proc Natl Acad Sci U S A 2016;113:14745-50.

2. Faergeman NJ, Knudsen J. Role of long-chain fatty acyl-CoA esters in the regulation of metabolism and in cell signalling. Biochem J 1997;323(Pt 1):1-12

3. Ellis JM, Bowman CE, Wolfgang MJ. Metabolic and tissue-specific regulation of acyl-CoA metabolism. PLoS One 2015;10:e0116587.

4. Jung SH, Lee HC, Hwang HJ, Park HA, Moon YA, Kim BC, et al. Acyl-CoA thioesterase 7 is involved in cell cycle progression via

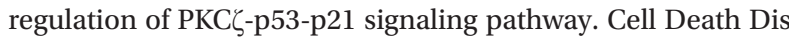
2017;8:e2793.

5. Ismael S, Purushothaman S, Harikrishnan VS, Nair RR. Ligand specific variation in cardiac response to stimulation of peroxisome proliferator-activated receptor-alpha in spontaneously hypertensive rat. Mol Cell Biochem 2015;406:173-82.

6. Wang F, Wu J, Qiu Z, Ge X, Liu X, Zhang C, et al. ACOT1 expression is associated with poor prognosis in gastric adenocarcinoma. Hum Pathol 2018;77:35-44.

7. Forwood JK, Thakur AS, Guncar G, Marfori M, Mouradov D, Meng W, et al. Structural basis for recruitment of tandem hotdog domains in acyl-CoA thioesterase 7 and its role in inflammation. Proc Natl Acad Sci U S A 2007;104:10382-7.

8. Yamada J. Long-chain acyl-CoA hydrolase in the brain. Amino Acids 2005;28:273-8.

9. Hunt MC, Greene S, Hultenby K, Svensson LT, Engberg S, Alexson SE. Alternative exon usage selectively determines both tissue distribution and subcellular localization of the acyl-CoA thioesterase 7 gene products. Cell Mol Life Sci 2007;64:1558-70.

10. Antoniotti S, Fiorio Pla A, Pregnolato S, Mottola A, Lovisolo D, Munaron L. Control of endothelial cell proliferation by calcium influx and arachidonic acid metabolism: a pharmacological approach. J Cell Physiol 2003;197:370-8.

11. Jenkins CM, Cedars A, Gross RW. Eicosanoid signalling pathways in the heart. Cardiovasc Res 2009;82:240-9.

12. Deng M, Brägelmann J, Schultze JL, Perner S. Web-TCGA: an online platform for integrated analysis of molecular cancer data sets. BMC Bioinformatics 2016;17:72.

13. Barrett T, Troup DB, Wilhite SE, Ledoux P, Rudnev D, Evangelista C, et al. NCBI GEO: mining tens of millions of expression profiles-database and tools update. Nucleic Acids Res 2007;35:D760-5.

14. Wright MW. A short guide to long non-coding RNA gene nomenclature. Hum Genomics 2014;8:7.

15. Kunej T, Obsteter J, Pogacar Z, Horvat S, Calin GA. The decalog of long non-coding RNA involvement in cancer diagnosis and monitoring. Crit Rev Clin Lab Sci 2014;51:344-57.

16. Engreitz JM, Haines JE, Perez EM, Munson G, Chen J, Kane M, et al. Local regulation of gene expression by lncRNA promoters, transcription and splicing. Nature 2016;539:452-5.

17. Feng X, Huang S. Effect and mechanism of long noncoding RNAs HOTAIR on occurrence and development of gastric cancer. J Cell Biochem 2019;120.

18. Fei ZH, Yu XJ, Zhou M, Su HF, Zheng Z, Xie CY. Upregulated expression of long non-coding RNA LINC00982 regulates cell proliferation and its clinical relevance in patients with gastric cancer. Tumour Biol 2016;37:1983-93.

19. Schmitt AM, Chang HY. Long noncoding RNAs in cancer pathways. Cancer Cell 2016;29:452-63.

20. Sheng Z, Wang X, Xu G, Shan G, Chen L. Analyses of a panel of transcripts identified from a small sample size and construction of rna networks in hepatocellular carcinoma. Front Genet 2019; 10:431.

21. Weber M, Schübeler D. Genomic patterns of DNA methylation: targets and function of an epigenetic mark. Curr Opin Cell Biol 
2007;19:273-80.

22. Steele N, Finn P, Brown R, Plumb JA. Combined inhibition of DNA methylation and histone acetylation enhances gene re-expression and drug sensitivity in vivo. Br J Cancer 2009;100:758-63.

23. Smith ZD, Meissner A. DNA methylation: roles in mammalian development. Nat Rev Genet 2013;14:204-20.

24. Klutstein M, Nejman D, Greenfield R, Cedar H. DNA methylation in cancer and aging. Cancer Res 2016;76:3446-50.

25. Livak KJ, Schmittgen TD. Analysis of relative gene expression data using real-time quantitative PCR and the 2(-Delta Delta C(T)) Method. Methods 2001;25:402-8.

26. Crane JD, Ogborn DI, Cupido C, Melov S, Hubbard A, Bourgeois JM, et al. Massage therapy attenuates inflammatory signaling after exercise-induced muscle damage. Sci Transl Med 2012;4:119ra13.

27. Pang G, Xie Q, Yao J. Mitofusin 2 inhibits bladder cancer cell proliferation and invasion via the Wnt/ $\beta$-catenin pathway. Oncol Lett 2019;18:2434-42.

28. Savan R, Chan T, Young HA. Lentiviral gene transduction in human and mouse NK cell lines. Methods Mol Biol 2010;612:209-21.

29. Zhou Z, Lin Z, Pang X, Tariq MA, Ao X, Li P, et al. Epigenetic regulation of long non-coding RNAs in gastric cancer. Oncotarget 2017; 9:19443-458.
30. Guo W, Dong Z, Shi Y, Liu S, Liang J, Guo Y, et al. Methylation-mediated downregulation of long noncoding RNA LOC100130476 in gastric cardia adenocarcinoma. Clin Exp Metastasis 2016;33:497508.

31. Laird PW. Oncogenic mechanisms mediated by DNA methylation. Mol Med Today 1997;3:223-9.

32. Van Tongelen A, Loriot A, De Smet C. Oncogenic roles of DNA hypomethylation through the activation of cancer-germline genes. Cancer Lett 2017;396:130-7.

33. Feinberg AP, Tycko B. The history of cancer epigenetics. Nat Rev Cancer 2004;4:143-53.

34. Wang D, Zhang Y, Huang Y, Li P, Wang M, Wu R, et al. Comparison of different normalization assumptions for analyses of DNA methylation data from the cancer genome. Gene 2012;506:36-42.

35. Kulis M, Esteller M. DNA methylation and cancer. Adv Genet 2010;70:27-56.

36. Gu Y, Chen T, Li G, Yu X, Lu Y, Wang H, et al. LncRNAs: emerging biomarkers in gastric cancer. Future Oncol 2015;11:2427-41.

37. Xuan Y, Wang Y. Long non-coding RNA SNHG3 promotes progression of gastric cancer by regulating neighboring MED18 gene methylation. Cell Death Dis 2019;10:694. 